\title{
Improving Eye Movement Control in Young Readers
}

\author{
TAINA M. LEHTIMÄKI* \& RONAN G. REILLY \\ Department of Computer Science, National University of Ireland Maynooth, \\ Maynooth, County Kildare, Ireland (*author for correspondence, e-mail: \\ taina@cs.nuim.ie)
}

\begin{abstract}
The objective of our study is to design and evaluate an oculomotor reading aid for beginning readers. The aid consists of an eye-tracking device and a computer program that gives real-time feedback in the form of a game to the subject about their fixation position on words. An experimental study was conducted with 8-year-old children. We evaluated the effectiveness of the aid for each child by comparing the landing site distributions before and after playing the game. We found that the peak of the landing site distribution moved towards the optimal viewing position (OVP) for word identification after playing the game. We also determined that training had a positive effect on gaze duration, on the mean and distribution of number of fixations per word, and on the percentage of words with refixations in the majority of subjects.
\end{abstract}

Keywords: children, eye movement control, optimal viewing position (OVP), reading, training

\section{Introduction and Motivation}

When people read, their eyes do not move smoothly along a line of text, but make fast movements called saccades and short stops called fixations. For an adult reading English, a typical saccade is 7-9 character positions and the typical fixation duration is 200 $300 \mathrm{~ms}$. Children's eye movement patterns differ in some ways from those of adults. Children make more refixations, more regressive (right to left) saccades, their saccades are shorter, fixations longer, and they fixate more often on the empty spaces (Buswell, 1922; Taylor, 1965).

Typically the initial saccades of experienced readers land at the centre or slightly left of the centre of a word at the position called the Preferred Viewing Location (McConkie et al. 1988). This tendency is beneficial to the reader since the optimal viewing position (OVP) for initial fixation is located near the centre of each word (O'Regan et al. 1984, 1987). O'Regan et al. found that when the eyes fixate at the OVP, word identification is faster and more accurate because: (i) the probability of refixation on the word is lowest, (ii) the gaze duration (the total time spent on a word) is shortest, and (iii) the time to 
identify the word is shortest. Also the probability of correct identification of a word is highest (O'Regan 1990).

However, during reading the eyes do not always work optimally; they frequently undershoot and overshoot the OVP of the word, and sometimes even the word boundary. This is especially true with beginning readers. The consequence is that extra fixations and/or movements back to previously read words need to be made. This leads to large reading time costs in the order of $200 \mathrm{~ms}$ per extra fixation (Blanchard, 1985). Current research has also shown that such extra fixations are usually triggered immediately on the basis of low-level visual processing. Furthermore, the extra refixations are often unnecessary; word recognition can often be achieved in a single fixation (Inhoff et al., 2000). An example of eye movement data collected in our study is shown in Figure 1. We have pointed out three typical eye movement patterns, which slow down the reading process especially with young readers.

McConkie et al. (1988) demonstrated that for adult readers the landing sites of saccades on a word are distributed as a Gaussian, where most fixations are located near the centre of the word. The location of the peak of the Gaussian curve and the standard deviation appeared to depend mainly on low-level oculomotor factors. For example, one of their findings was that when the launch site (the location of the prior fixation) is further to the left of the target word, the centre of the Gaussian systematically shifts leftward.

The question arises whether training can influence these low-level processes. The objective of our study was to design and evaluate an oculomotor reading aid for beginning readers. The aid consists of an

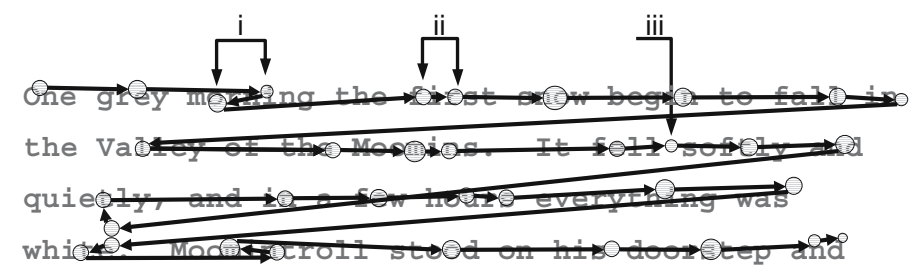

Figure 1. This is sample data from our experimental study, where the circles represent fixations, the size of each circle represents the duration of that fixation, and the arrows represent saccades. We point out three typical eye movement patterns, which slow down the reading process: (i) initial fixation at the end of the word followed by regressive (right to left) saccade, (ii) initial fixation at the beginning of the word followed with refixation at centre of the word, and (iii) fixation on the empty space between the words. 
eye-tracking device and a computer program, the OVP game, which gives real-time feedback to the subject about their fixation position. The goal is to teach the readers to land their saccades at the OVP of each word. We use the oculomotor reading aid for an experimental study with 8-year-old children. We evaluate the effectiveness of the aid for each child by comparing the landing site distributions fitted with Gaussian curves before and after playing the game. We also determine if training has had an effect on gaze duration, mean number of fixations per word, and percentage of words with refixations.

The paper is organised as follows. In Section 2, we describe the OVP game training program. In Section 3, we outline the experimental study for evaluating the effects of the reading aid. The results and analysis are provided in Section 4, followed by discussion and conclusions in Section 5.

\section{The OVP Game}

The idea of the training is to teach a reader to control their eye movements during reading so that the saccades they make would land at the centre of the word (at the OVP). Eye movement control during reading is a highly automated process; people are not aware of most of the saccades and fixations they make. We wanted to find a way to train children to control these subconscious saccades and fixations. We wanted to make the training as interesting as possible for them, so we decided to do it in the form of a computer game.

The basic idea of the OVP game is to follow a reader's eye movements in real time with an eye-tracking system and give them feedback according to their fixation location. We built our game to work with the EyeLink II (www.eyelinkinfo.com) eye-tracking system. This consists of three miniature cameras mounted on a headband. Two eye cameras allow binocular eye tracking and the third camera tracks the orientation of the head with respect to the computer screen. The system permits the subject to make small natural head movements, which is very important feature when working with children.

The game consists of six screens of word lists. The letters are masked with x's so all that the participant sees is a screen filled with strings of x's (see Figure 2). The actual word is revealed when the participant fixates somewhere in the central third of the word. Fixating in the first third of the word, or in the final third of the word does not have any effect, so in order to reveal the words the reader has to 


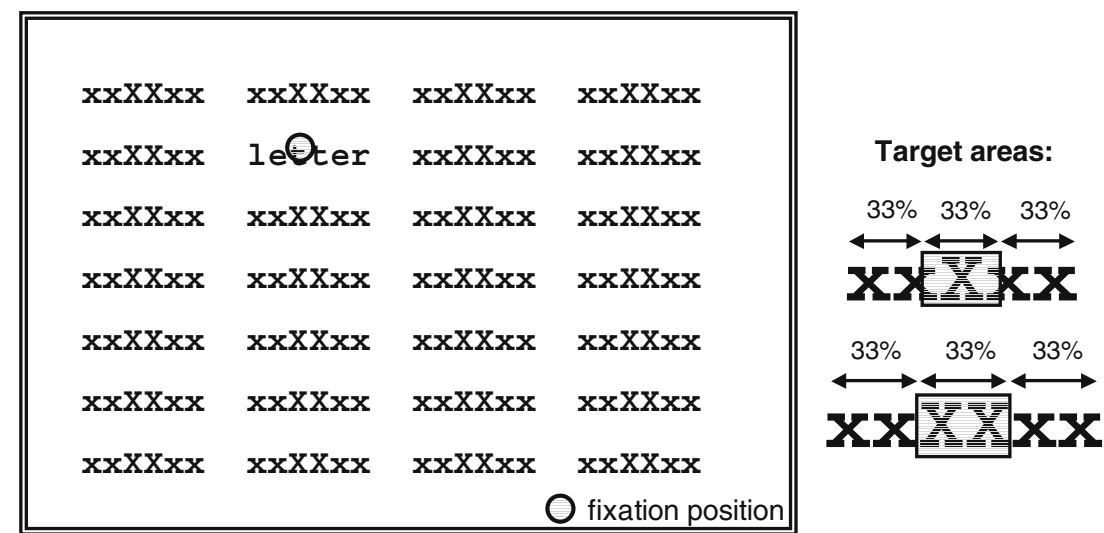

Figure 2. A sample screen from the OVP game. The participant reveals the word by fixating at its centre. The width of target area is $33 \%$ of the width of the word.

fixate approximately at the OVP. The timing threshold on the revealing of the word is set so that saccades crossing the centre of the word will not reveal the word; the reader must actually fixate at the centre. This comprises the real-time feedback to the subject about their fixation position. The task for the participant is to reveal as many words as possible in $60 \mathrm{sec}$. After the initial testing we decided to add visual clues at the centre to help the reader to target the fixations. We added capital X's at the OVPs ( $\mathrm{xxXxx}$ for five-letter words and $\mathrm{xxXXxx}$ for six-letter words) for the first four screens of the game. The game gets more difficult from screen to screen; from 28 to 104 words per screen. The last two screens are without capital X's at the OVPs. For the control group, the OVP game was different. The target area for revealing the words was the whole word, instead of the centre.

\section{Experimental Study}

\subsection{Method and participants}

We evaluated the effects of playing the OVP game by doing an experimental study with a group of local school children. Subjects were 8 -year-old boys, who had average reading skills. The experiments were performed during the last month of their school year and during school hours. We originally had a group of 13 participants, but due to 
the calibration difficulties we ran the experiments with only five subjects and two control subjects.

Each session consisted of $10 \mathrm{~min}$ of data collection before the game, $10 \mathrm{~min}$ of training, and $10 \mathrm{~min}$ of data collection after the game. These times include the calibrations. Our aim was to have three sessions per child, but for one of the subjects and one of the controls we only had two sessions. The subjects had between one and seven days between their sessions, depending on their school schedule.

We recorded eye movement data before and after playing the OVP game. The stimuli were a list of five-letter words, a list of six-letter words, and four screens of continuous text. In the word lists there were 91 five-letter words and 78 six-letter words. For the text we choose part of an age-appropriate children's novel [Finn Family Moomintroll by Tove Jansson (1948)]. These stimuli were presented in 13 double spaced lines in a typeface with fixed-width characters. When analysing the data, the data recorded from the first and last lines of each screen, and from the first and the last word of each line, were not used. Furthermore, for the continuous text, we chose only the fiveand six-letter words in the text in the analysis.

Children were advised to read silently, and to try to read each word. If they did not know the word, they were advised to just continue reading. We also explained that this was not a reading test or contest in order to make them feel more comfortable about the situation. They were introduced to the characters from the book before starting the experiment. This was done also to help make them more relaxed. Although the EyeLink II eye-tracking system is able to handle small head movements, we had a chin rest for the subjects to encourage them to keep their heads as still as possible. We also used a silicon swimming cap under the headband to keep it from slipping. We used only the pupil-tracking mode of the EyeLink II system, since the corneal reflection mode did not calibrate well on children.

We ran a calibration routine displaying individually nine calibration points, spread evenly across the computer screen. The subject was advised to look in the centre of the calibration point and the fixation position was recorded for each of these points. Calibration was done at the beginning of the data collection stages and at the beginning of the game. For most subjects extra calibrations were needed during the experiments due to headband slippage. 


\section{Results and Analysis}

In this section, we describe the results of the experimental study. We first analyse how playing the OVP game changes the landing site distributions of the subjects when reading 5-letter words. Next we analyse the changes in gaze duration, and the mean value of the number of fixations per word. This is done for the five- and six-letter words and the continuous text. Finally we have in-depth analysis of how the number of fixations actually changes for five-letter words. Due to significant individual differences we describe the results individually for each subject.

\subsection{Change in landing site distributions}

The landing site distribution of the initial fixations for five-letter words for each subject is presented in Figure 3. The plots show data collected before and after playing the OVP game. Only landing site data for reading a word for the first time is included. Figures combine initial fixations from all training sessions the subject attended (Subject $A$ did not attend to session three). The vertical axis represents the number of initial fixations. The horizontal axis represents the landing
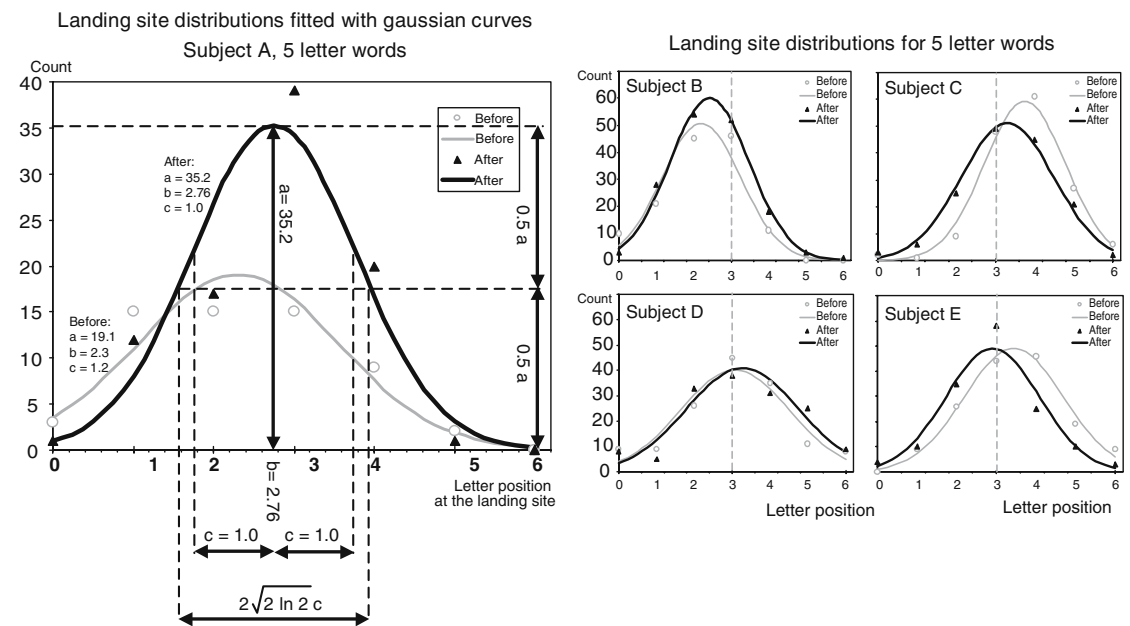

Figure 3. Landing site distributions and fitted Gaussian curves for five-letter words before and after playing the OVP game. The landing site distribution for Subject A also illustrates graphically the Gaussian parameters: $a$ is amplitude (the height of the curve), $b$ is mean (the location of the peak on the horizontal axis) and $c$ is standard deviation (how wide the curve is). 
Table 1. The numerical values for fitting the Gaussian curves for all subjects: amplitude $(a)$, mean $(b)$, standard deviation $(c)$, and the sum of squared residuals

\begin{tabular}{|c|c|c|c|c|c|c|c|c|c|c|}
\hline & \multicolumn{2}{|c|}{ Subject A } & \multicolumn{2}{|c|}{ Subject B } & \multicolumn{2}{|c|}{ Subject C } & \multicolumn{2}{|c|}{ Subject D } & \multicolumn{2}{|c|}{ Subject E } \\
\hline & Before & After & Before & After & Before & After & Before & After & Before & After \\
\hline$a$ & 19.05 & 35.20 & 50.70 & 60.00 & 59.25 & 51.20 & 40.20 & 40.90 & 49.15 & 49.14 \\
\hline$b$ & 2.31 & 2.76 & 2.20 & 2.42 & 3.75 & 3.31 & 3.07 & 3.28 & 3.44 & 2.91 \\
\hline$c$ & 1.25 & 1.02 & 1.05 & 1.06 & 1.04 & 1.18 & 1.44 & 1.48 & 1.25 & 1.18 \\
\hline $\begin{array}{l}\text { Sum of } \\
\text { square } \\
\text { residuals }\end{array}$ & 0.00 & 0.05 & 0.07 & -0.08 & 0.01 & 0.03 & 0.09 & -0.03 & 0.01 & 0.02 \\
\hline
\end{tabular}

site showing the letter positions where the initial fixations landed: zero meaning the space before the word, one meaning the first letter, and so on, with six representing the space after the word. The optimal landing site for five-letter words would be the letter position three. We use McConkie's finding to evaluate the landing site distributions by fitting the raw data with Gaussian curves (McConkie et al., 1988). We use the Gaussian distribution function

$$
y(x)=a \exp \left[\left(-(x-b)^{2}\right) /\left(2 c^{2}\right)\right]
$$

where $x$ is the landing site position within the word, $a$ is the amplitude, $b$ is the mean, and $c$ is the standard deviation. These Gaussian parameters are graphically explained in Figure 3 with the graph of Subject A's data after playing the OVP game. The numerical values for parameters $a, b$ and $c$ for all subjects are presented in Table 1 .

For fitting the Gaussian parameters $b$ and $c$, we used the statistical values of mean and standard deviation of the observed data. For getting suitable values for the parameter $a$, we wanted our observed data points to spread evenly above and below the fitted Gaussian curve. To obtain this we set the parameter $a$ so that the sum of the residuals (observed value subtracted from the expected value) was as close as possible to zero; we accepted values between -0.1 and 0.1 . The sums of residuals for each subject are also presented in Table 1.

From the graphs in Figure 3, we can see that the amplitude gets clearly larger for the Subjects A and B, does not appear to change for Subjects D and E, and gets smaller for Subject C. For the mean landing site location we notice a clear shift towards the optimal position (the letter position three) on Subjects A and C. The shift is in 
the desired direction, but not large for Subjects B and E. There is no desirable shift for Subject D. A desirable change in the standard deviation is present only for Subject A, and there is an opposite change for Subject $\mathrm{C}$. The most important changes with which to evaluate the effects of the training are the changes in the mean value. We can conclude that the training game had clear desired effect on the landing site distribution curve for Subject A. The effect for Subjects B, C and $\mathrm{E}$ were in an acceptable direction, but not as clearcut as for A. There was no effect for Subject D.

\subsection{Change in gaze duration}

The results for the changes in gaze duration are provided in Figure 4. The vertical axis denotes gaze duration time in milliseconds, and the horizontal axis is divided into sections according to subject, session number, and stimuli type (five-letter words, six-letter words and continuous text). The changes in gaze durations are clearly visible with the bars at the bottom of the graph. If the bar is above the horizontal axis, the change is desirable, meaning that the gaze duration after playing the game is shorter than before the game. For Subjects A, B and $\mathrm{C}$ the changes in gaze durations seem to be as desired. For Subject $\mathrm{D}$ the first session seemed to have a good effect, but the later

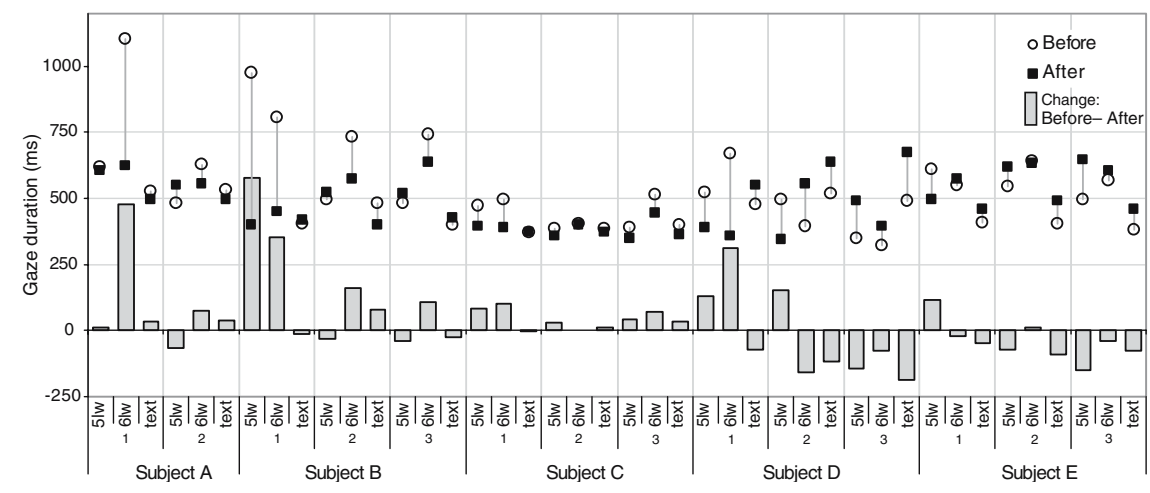

Figure 4. Gaze durations before and after playing the training game are marked with circles and squares, respectively. On the bottom of the figure the change in gaze duration is visualised with bars (a bar above the horizontal axis means the gaze duration was shortened due to playing the OVP game - the desirable outcome). The horizontal axis is divided into sections according to subject (A-E), session number (1-3), and stimuli type ('5lw' means five-letter words, '6lw' means six-letter words, and 'text' means continuous text). 
sessions did not. For the Subject $\mathrm{E}$ the gaze durations after the game were longer than before the game. For the different stimuli types our results show the training did shorten the gaze duration most when reading the word lists; however, there was no observable change to gaze durations for subjects reading continuous text.

\subsection{Change in mean number of fixations per word}

In Figure 5, we plot changes in the mean value of number of fixations per word. The data is presented in similar way to that in Figure 4: the horizontal axis is identical but the vertical axis shows now the number of fixations. The change is desirable if the bars at the bottom are above the horizontal axis. From this plot we observe that the general tendency is that fewer fixations are needed after playing the game. For example, before playing the game Subject A needed an average 4.3 fixations to read a five-letter word, and after playing the game the average had dropped to 2.5. However, Subjects D and E only got a benefit from the first session. For the different stimulus types the training seemed to work best for reducing the fixations when reading the lists of words, and the effects in reading continuous text were less evident. The similarity between Figures 4 and 5 suggests, as expected, that reducing the number of fixations also reduces the gaze durations thereby making the reading process faster.

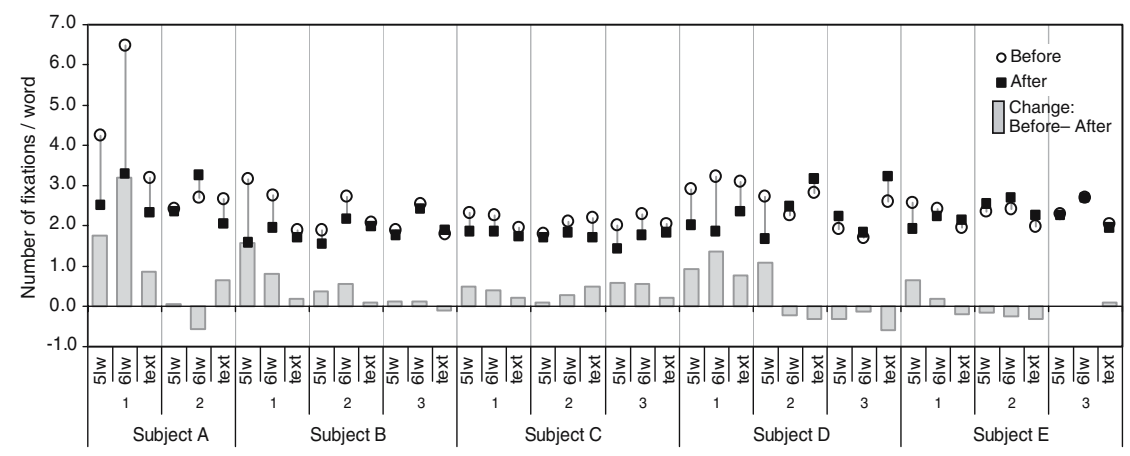

Figure 5. Mean number of fixations per word before and after subjects played the training game, are marked with circles and squares, respectively. The bars at the bottom of the figure show the change. A bar above the horizontal axis means the number of fixations per word is smaller after the training - the desirable outcome. The horizontal axis is divided into sections according to subject (A-E), session number (1-3), and stimuli type ('5lw' means five-letter words ' $6 \mathrm{lw}$ ' means six-letter words, and 'text' means continuous text). 


\subsection{Distribution of number of fixations per word}

Figure 6 shows an in-depth analysis of how the number of fixations changed due to training for each subject. Here, we present a distribution of the number of fixations for five-letter words for each session. It is very clear how at the beginning of session 1 Subjects A and B have an almost flat distribution of number of fixations per word. After playing the game, for Subject A most words are read with two fixations, and Subject B is able to read most words with a single fixation. There is a suggestion that the tendency to read words with less fixations could carry on to the next session; the 'before' distribution for session 2 resembles closely the 'after' distribution of session 1 for both Subjects A and B. However, our small study does not provide enough evidence to make this claim convincingly. For Subjects C and D, training improved their distribution curves, but the effect does not seem to carry over between sessions in their cases. For Subject E first session shows improvement, but the other two are showing a negative effect.

\section{Discussion and Conclusions}

We have presented the results of an experimental study to evaluate an oculomotor reading aid for beginning readers. The aid consists of an

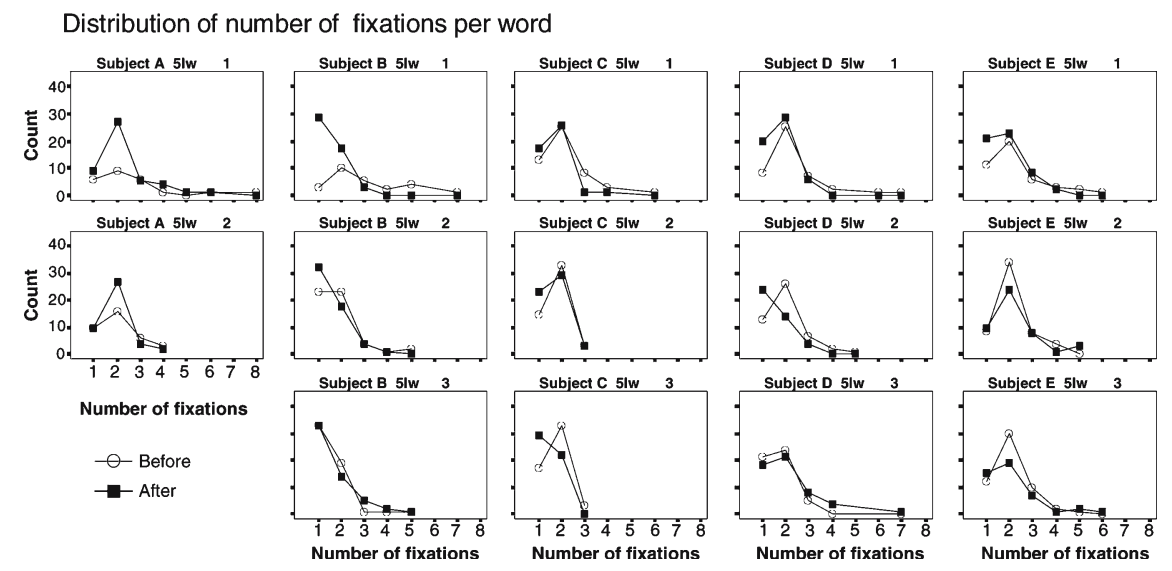

Figure 6. Distribution of number of fixations per word before and after the game for reading five-letter words. White circles are used to mark data collected before the training, and black squares are for after. The first row of graphs is for session 1 , the second for session 2 and third for session 3. Subject A did not attend session 3. 
eye-tracking device and a computer program, an OVP game, that gives real-time feedback to the subject about their fixation position. The experimental study was conducted with 8-year-old children. Working with children was quite challenging. We faced some difficulties during the experiments. For some subjects the headband of the eye-tracking system felt uncomfortable and could not be tightened properly due to their small head size. This caused the headband to slip easily. Using a silicon swimming cap under the headband reduced the slippages significantly. Subjects also sometimes started to talk or read aloud during the experiment. This caused large head movements due to their chin being on a chin rest. For some subjects the sessions lasted up to $60 \mathrm{~min}$ instead of 30 , since many recalibrations were needed, and due to breaks requested by the subject in question. Furthermore, the longer the session went the more difficult it was for the children to sit still. This happened especially with Subject E, who found the headband and chinrest very uncomfortable, and had to move his head several times during the experiment. Subject D was quite afraid of the experimental situation and was very nervous, which could explain why playing the game did not have positive effect on his reading performance.

We evaluated the effectiveness of the aid for each child by comparing the landing site distributions before and after playing the game. We can conclude that the training game had the desired effect on the landing site distribution curve for the Subject A. For the Subjects B, $\mathrm{C}$ and $\mathrm{E}$ the training had some effect towards the desired direction, although the effect was less pronounced. The training did not have desired effect for Subject D. The most important improvement was that the peak of the landing site distribution moved towards the OVP for four subjects. We have also determined that training had a desirable effect on gaze duration, mean number of fixations per word, and distribution of number of fixations per word in the majority of subjects.

For the different stimuli types our results show that although the training had a positive effect when reading the word lists, the training did not improve the reading of continuous text. Reading lists of words of fixed length require subjects to make a series of saccades of equal size, however in normal continuous reading the saccade lengths vary. To create an appropriate oculomotor reading aid for reading continuous text, it would be important to vary the saccade lengths during training. 
Where there is a positive effect of the training game, it is possible that the effect lasts over days, and not just minutes, as shown by Figure 6. This however could also be due to familiarity with the routine.

In this study, we were able to show that it is possible to train people to control their eye movements during reading. This suggests that developing an oculomotor reading aid is an acceptable idea. In this study we only had five subjects. Firm conclusions are difficult to draw because the data set was small, but the results are encouraging enough to warrant further research.

\section{Acknowledgements}

We would like to thank the staff and pupils of Maynooth Boys National School for their cooperation with this study.

\section{References}

Blanchard, H. E. (1985). A Comparison of Some Processing Time Measures Based on Eye Movements. Acta Psychologica 58: 1-15.

Buswell, G. T. (1922). Fundamental Reading Habits: A Study of Their Development. University of Chicago Press: Chicago.

Inhoff, A., Radach, R. \& Heller, D. (2000). Complex Compounds in German: Interword Spaces Facilitate Segmentation but Hinder Assignment of Meaning. Journal of Memory and Language 42: 23-50.

McConkie, G. W., Kerr, P. W., Reddix, M. D. \& Zola, D. (1988). Eye Movement Control During Reading: I. The Location of Initial Eye Fixations on Words. Vision Research 28(10): 1107-1118.

O’Regan, J. K., Lévy-Schoen, A., Pynte, J. \& Brugaillère, B. (1984). Convinient Fixation Location Within Isolated Words of Different Length and Structure. Journal of Experimental Psychology: Human Perception and Performance 10: 250-257.

O'Regan, J. K. \& Lévy-Schoen, A. (1987). Eye Movement Strategy and Tactics in Word Recognition and Reading. In Coltheart, M. (ed.) Attention and Performance XII: The Psychology of Reading, 363-383. Erlbaum: Hillsdale, NJ.

O'Regan, J. K. (1990). Eye Movements and Reading. In Kowler, E. Eye Movements and Their Role in Visual and Cognitive Processes, 395-435. Elsevier Science Publishers: Amsterdam.

Taylor, E. A. (1965). The Fundamental Reading Skill. C.C Thomas: Springfield, Il. 